\title{
The Impact of the Covid-19 Pandemic with Hidradenitis suppurativa Being Treated with Adalimumab: Evidence from Real-World Data
}

\section{Turkmen $\mathrm{M}^{*}$ and Dogan $\mathrm{S}$}

Dermatology Department, Izmir Bozyaka Training and Research Hospital, Turkey

*Corresponding author: Meltem Turkmen, MD, Izmir Bozyaka Training and Research Hospital, Dermatology Department, Izmir, Turkey, Tel: +905538488826; Email: meltemturkmen@ hotmail.com

\section{Research Article}

Volume 5 Issue 4

Received Date: September 29, 2020

Published Date: October 16, 2020

DOI: $10.23880 /$ cdoaj-16000222

\section{Abstract}

Background: There is uncertainty concerning the outcome of coronavirus disease-19 (COVID-19) infections in patients who are receiving adalimumab for hidradenitis suppurativa (HS) treatment. Objectives: This study aims to investigate the effect of adalimumab in HS patients during COVID 19 outbreak.

Methods: We performed a retrospective observational analysis of the moderate-to-severe HS patients under adalimumab therapy, with reporting the number of patients hospitalized or who died from COVID 19 infection between 13 March 2020 and 31 July 2020.

Results: Eighty nine patients who continued their therapy during pandemic were evaluated retrospectively. There were no cases of death from COVID-related disease in our study population or patients hospitalized for COVID-related disease.

Conclusions: We suggest continuing the treatment of ADA in HS patients, under COVID-19 pandemic.

Keywords: COVID-19; Hidradenitissuppurativa; Adalimumab

\section{Introduction}

Severe acute respiratory syndrome coronavirus 2 has spread over all over the world, causing the respiratory manifestations of coronavirus disease-19 (COVID-19) and satisfying the epidemiological criteria for a pandemic [1]. Turkey has been one of the most affected areas in Europe.

Hidradenitis suppurativa (HS) is a chronic, recurrent, inflammatory dermatosis affecting the follicular unit. It is characterized by abscesses, nodules and sinus tracts, as well as scarring predominantly in the axillary and groin regions [2-4]. HS is characterized by immune dysregulation. The studies have indicated that HS patients have higher levels of proinflammatory cytokines tumor necrosis factor $\boldsymbol{\alpha}$ (TNF- $\boldsymbol{\alpha})$,

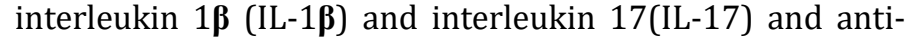

inflammatory cytokine interleukin 10 (IL10) in their serum and affected skin lesions [5-7]. Adalimumab (ADA) is a recombinant human IgG1 monoclonal antibody that binds and blocks the activity of TNF- $\alpha$ expressed in hair follicles and sweat glands [8]. ADA is the only approved drug for the treatment of moderate-to-severe HS by the Food and Drug Administration (FDA) and the European Medicines Agency (EMA) [9-11]. It is unclear that this immunosuppressive/ immunomodulatory agent increases the risk of severe COVID 19 infection. In addition HS patients tend to have multiple medical comorbidities, including smoking history, cardiovascular diseases, obesity, diabetes, metabolic syndrome, depression, dyslipidemia, leading to overall poorer health which are also risk factors for severe COVID-19 infection $[12,13]$. 


\section{Clinical Dermatology Open Access Journal}

Thus, we performed this study aiming to understand the effect of adalimumab in HS patients during COVID 19 outbreak.

\section{Materials and Methods}

We performed a retrospective observational analysis of the moderate-to-severe HS patients under adalimumab therapy, with reporting the number of patients hospitalized or who died from COVID 19 infection between 13 March 2020 and 31 July 2020 . We evaluated the demographic features and clinical data of patients (age, gender, Hurley Stage, comorbidities) by consulting electronic medical records of patients or by contacting patients through phone call or visit. We excluded the patients who were in the induction phase or who autonomously discontinued the therapy at the start of the pandemic.

\section{Results}

Ninety two moderate-to-severe HS patients were being treated with adalimumab in our clinic and three of them autonomously stopped the therapy at the beginning of the pandemic. Eighty nine patients who continued their therapy were evaluated retrospectively. The ages of 89 patients were between 19-67 (mean: $47+/-14,3$ ) and $65(73,03 \%$ ) of them were male. Duration of adalimumab therapy ranged from 1 to 7 years (mean 4,3+/- 2,4years). Twenty two of the patients according to the Hurley classification were determined as stage II and 67 as stage III. Metabolic syndrome were reported in 20,22 \% of patients while cardiovascular diseases, hypertension, diabetes mellitus and chronic obstructive pulmonary disease was reported in $23,59 \%$, $31,46 \%, 21,34 \%, 7,86 \%$ of patients, respectively. One of our patients, a 36 year-old man, was diagnosed with HIV infection in 2011. He was suffering from severe axillary and inguinal hidradenitis suppurativa, Hurley stage III, for 8 years and has been under adalimumab treatment for a year. His CD4 count at the beginning of the pandemic was 452cells/ $\mathrm{mm} 3$ and viral load was is undetectable under abacavir with lamivudine and rilpivirin therapy.

There were no cases of death from COVID-related disease in our study population or patients hospitalized for COVIDrelated disease. Characteristics of the patients are presented in Table 1.

\begin{tabular}{|c|c|}
\hline Number of patients with HS & 89 \\
\hline Female, $\mathrm{n}(\%)$ & $24(26,96)$ \\
\hline Male, n (\%) & $65(73,03)$ \\
\hline Age, years (mean +/-SD) & $47+/-14,3$ \\
\hline BMI, $\mathrm{kg} / \mathrm{m}^{2}($ mean +/- SD) & $27,9+/-2,5$ \\
\hline \multicolumn{2}{|c|}{ Hurley Stage, $\mathrm{n}(\%)$} \\
\hline II & $22(24,71)$ \\
\hline III & $67(75,28)$ \\
\hline Duration of adalimumab therapy, years (mean $+/-\mathrm{SD}$ ) & $4,3+/-2,4$ \\
\hline Smoking, n (\%) & $71(79,77)$ \\
\hline \multicolumn{2}{|c|}{ Comorbidity, n (\%) } \\
\hline Metabolic syndrome & $18(20,22)$ \\
\hline Cardiovascular disease & $21(23,59)$ \\
\hline Hypertension & $28(31,46)$ \\
\hline Diabetes mellitus & $19(21,34)$ \\
\hline Chronic obstructive pulmonary disease & $7(7,86)$ \\
\hline Acquired Immune Deficiency Syndrome & $1(1,12)$ \\
\hline \multicolumn{2}{|c|}{$\begin{array}{l}\text { Outcome measure, } n \\
\end{array}$} \\
\hline Hospitalized for COVID-related disease & 0 \\
\hline Deaths from COVID-related disease & 0 \\
\hline
\end{tabular}

Table 1: Characteristics of patients with HS treated with adalimumab therapy. 


\section{Clinical Dermatology Open Access Journal}

\section{Discussion}

Biologics, as previously suggested by both trials and real-life registries, increased airway infections and there is a concern that patients on biologic treatment may be at risk because of COVID infection [14]. However, recent articles did not support this conclusion $[15,16]$. On the other hand, their inhibition of pro-inflammatory cytokines in the viral phase which is characterized by an exaggerated immune response (cytokine storm) with high TNF- $\alpha$ levels, might even be protective [17].

Rozzo G.et al performed a retrospective observational analysis of the 96 moderate and severe HS patients under systemic treatment in the pandemic, out of them $47,9 \%$ were under adalimumab treatment [18]. In their study there were no cases of death or hospitalization because of COVID-19 infection. Marasca C, et al. reported the experience of $93 \mathrm{HS}$ patients followed by telephone-based consultations and $80 \%$ of them were under adalimumab therapy. None of them were diagnosed as COVID-19 infection and there were no cases of death or hospitalization because of COVID-19 infection [19]. They suggested to continue the ongoing treatment unless symptoms such as fever, cough and dyspnea occurred [19].

Clinical course and outcome of COVID-19 among patients with HIV-1 infection are still unknown. Some authors have suggested an increased risk for COVID-19 due to HIV-1-related immunosuppression [20]. On the other hand, some authors speculated that HIV-1-infected patients on combination antiretroviral therapy (cART) may have a lower risk for COVID-19 and related complications because of the in vitro activity of some antiretroviral drugs against COVID-19 infection [21,22]. In our study one of our patients had HIV-1 infection and he continued both his cART and adalimumab therapy. He has not experience the symptoms of COVID-19 infection like fewer, cough and dyspnea.

We acknowledge the limitations of this retrospective observational study which include the absence of molecular or serological investigations for the diagnosis of COVID-19 infection in the study population. However, the objective of this study was not to investigate the COVID-19 infections in HS patients, but to report the occurrence of hospitalization or death, as indicators of severe outcomes related to COVID-19 infection. Patients with HS tend to have multiple medical comorbidities, including cardiovascular diseases, diabetes, metabolic syndrome, leading to overall poorer health and, above all, treated with ADA (an immunosuppressive and immunomodulation agent), there were no cases of hospitalization or death from COVID-19. In our study population no patient has reported any symptoms of COVID-19 infection. We suggest continuing the treatment of ADA in HS patients, under COVID-19 pandemic.

\section{References}

1. Callaway E (2020) Time to use the p-word? Coronavirus enters a dangerous new phase. Nature 579: 12.

2. Sellheyer K, Krahl D (2005) "Hidradenitis suppurativa" is acne inversa! An appeal to (nally) abandon a misnomer. Int J Dermatol 44(7): 535-540.

3. Jemec GB (2012) Clinical practice. Hidradenitis suppurativa. N Engl J Med 366(2): 158-164.

4. Fimmel S, Zouboulis CC (2010) Comorbidities of hidradenitis suppurativa (acne inversa). Dermatoendocrinol 2(1): 9-16.

5. Porter ML, Golbari NM, Lockwood SJ, Kimball AB (2018) Overview and update on biologic therapy for the moderate-to-severe hidradenitis suppurativa. Semin Cutan Med Surg 37(3): 182-189.

6. Van der Zee HH, de Ruiter L, van den Broecke DG, Dik WA, Laman JD, et al. (2011) Elevated levels of tumour necrosis factor (TNF)- $\alpha$, interleukin (IL)-1 $\beta$ and IL-10 in hidradenitis suppurativa skin: a rationale for targeting TNF- $\alpha$ and IL-1 $\beta$. Br J Dermatol 164(6): 1292-1298.

7. Kimball AB, Okun MM, Williams DA, Gottlieb Ab, Papp KA, et al. (2016) Two Phase 3 Trials of Adalimumab for Hidradenitis suppurativa. N Engl J Med 375(5): 422-434.

8. Zouboulis CC, Desai N, Emtestam L, Hunger RE, Ioannides D, et al. (2015) European S1 guideline for the treatment of hidradenitis suppurativa/acne inversa. J Eur Acad Dermatol Venereol 29(4): 619-644.

9. Kimball AB, Kerdel F, Adams D (2012) Adalimumab for the treatment of moderate to severe Hidradenitis suppurativa: a parallel randomized trial. Ann Intern Med 157(12): 846-855.

10. Kimball AB, Sobell JM, Zouboulis CC, Gu Y, Williams DA, et al. (2016) HiSCR (Hidradenitis suppurativa Clinical Response): a novel clinical endpoint to evaluate therapeutic outcomes in patients with hidradenitis suppurativa from the placebo-controlled portion of a phase 2 adalimumab study. J Eur Acad Dermatol Venereol 30(6): 989-994.

11. Zouboulis CC, Okun MM, Prens EP, et al. (2019) Longterm adalimumab efficacy in patients with moderate-tosevere hidradenitis suppurativa/acne inversa: 3-year results of a phase 3 open-label extension study. J Am Acad Dermatol 80(1): 60-69.

12. Tzellos T, Zouboulis CC (2020) Review of comorbidities of hidradenitis suppurativa: implications for daily clinical 


\section{Clinical Dermatology Open Access Journal}

practice. Dermatol Ther (Heidelb) 10(1): 63-71.

13. Zheng Z, Peng F, Xu B, Zhao J, Liu H, et al. (2020) Risk factors of critical \& mortal COVID-19 cases: a systematic literature review and meta-analysis. J Infect 81(2): 16-25.

14. Conforti C, Giuffrida R, Dianzani C, Meo ND, Zalaudek I (2020) COVID-19 and psoriasis: is it time to limit treatment with immunosup pressants? A call for action. Dermatol Ther 11: 13298.

15. Bardazzi F, Loi C, Sacchelli L, Amber AD (2020) Biologic therapy for psoriasis during the covid-19 outbreak is not a choice. J Dermatolog Treat 31(4): 320-321.

16. Megna M, Ruggiero A, Marasca C, Fabbrocini G (2020) Biologics for psoriasis patients in the COVID-19 era: more evidence, less fears. J Dermatolog Treat 31(4): 1-2.

17. Siddiqi HK, Mehra MR (202) COVID-19 illness in native and immunosuppressed states: a clinical-therapeutic staging proposal. J Heart Lung Transpl 39(5): 405-407.
18. Rozzo G, Ramondetta A, Fierro MT, Dapavo P, Ribero S (2020) Moderate-to-severe hidradenitis suppurativa under systemic therapy during the COVID-19 outbreak. Dermatol Ther 33(4): e13680.

19. Mascara C, Ruggiero A, Megna M, Annunziata MC, Fabbrocini G (2020) Biologics for patients affected by hidradenitis suppurativa in the COVID-19 era: data from a referral center of Southern Italy. J Dermatolog Treat 25.

20. Dauby N (2020) Potential impact of COVID-19 in people living with HIV: experience from previous 21st century coronaviruses epidemics. AIDS 34(8): 1255-1256.

21. Toombs JM, Van den Abbeele K, Democratis J, Merricks R, Mandal AKJ, et al. (2020) COVID-19 in 3 people living with HIV in the United Kingdom. J Med Virol: 10.

22. Mascolo S, Romanelli A, Carleo MA, Esposito V (2020) Could HIV infection alter the clinical course of SARSCoV-2 infection? When less is better. J Med Virol 92(10): 1777-1778. 